\title{
Deficiencies in Chfr and MIh1 synergistically enhance tumor susceptibility in mice
}

Zheng Fu, ${ }^{1}$ Kevin Regan, ${ }^{1}$ Lizhi Zhang, ${ }^{2}$ Michael H. Muders, ${ }^{3}$ Stephen N. Thibodeau, ${ }^{2}$ Amy French, ${ }^{2}$ Yanhong Wu, ${ }^{2}$ Scott $\mathrm{H}$. Kaufmann, ${ }^{4}$ Wilma L. Lingle, ${ }^{2}$ Junjie Chen, ${ }^{5}$ and Donald J. Tindall1

\begin{abstract}
1Department of Urology and Department of Biochemistry and Molecular Biology, Mayo Clinic College of Medicine, Rochester, Minnesota, USA
2Department of Laboratory Medicine and Pathology, Mayo Clinic College of Medicine, Rochester, Minnesota, USA. ${ }^{3}$ Institute of Pathology, University Hospital Carl-Gustav-Carus, Dresden, Germany. ${ }^{4}$ Department of Oncology, Mayo Clinic College of Medicine, Rochester, Minnesota, USA. 5Department of Therapeutic Radiology, Yale University School of Medicine, New Haven, Connecticut, USA.
\end{abstract}

\begin{abstract}
Genetic instability, which leads to an accumulation of various genetic abnormalities, has been considered an essential component of the human neoplasic transformation process. However, the molecular basis of genomic instability during tumorigenesis remains incompletely understood. Growing evidence indicates that checkpoint with forkhead and ring finger domains (CHFR), a recently identified mitotic checkpoint protein, plays an important role in maintaining chromosome integrity and functions as a tumor suppressor. In this study, we used high-throughput technology to conduct gene expression profiling of human colon cancers and found that loss of CHFR expression frequently occurred in colon cancers with high microsatellite instability (MSI-H). Downregulation of CHFR expression was closely associated with overexpression of Aurora A, an important mitotic kinase. Mice with deficiencies in both Chfr and Mlb1 (the gene that encodes the DNA mismatch-repair protein Mlh1) displayed dramatically higher incidence of spontaneous tumors relative to mice deficient for only one of these genes. These results suggest that defects in both Chfr and Mlb1 synergistically increase predisposition to tumorigenesis.
\end{abstract}

\section{Introduction}

Genetic instability is a hallmark of human tumors. Current evidence indicates the existence of 2 major types of genomic instability: microsatellite instability (MSI) and chromosomal instability (CIN) (1). MSI is the phenotypic consequence of a deficient DNA mismatch-repair (MMR) system. MMR enzymes, which belong to an evolutionary conserved family of DNA repair proteins, normally repair replication errors generated by DNA polymerases during DNA replication. The bacterial MutS detects the mismatched DNA, whereas MutL creates nicks in the DNA, marking it for repair. In humans, the MutS homologs are MSH2 (MutS homolog 2), MSH3, and MSH6, and the MutL homologs are MLH1 (MutL homolog 1), PMS1, and PMS2. The inactivation of MMR genes such as MLH1 or MSH2 in tumors gives rise to genomic instability at the nucleotide sequence level, which can be most easily detected as changes at short sequences of DNA repeats (microsatellites) scattered throughout the genome. Cancer cells with this MSI have nucleotide mutation rates 2 to 3 orders of magnitude greater than those observed in normal cells or MMR-proficient cancers of the same cell type (2-4). MSI is observed in a substantial fraction of colorectal cancers (CRCs) from patients with hereditary nonpolyposis CRC (HNPCC) (5), approximately $15 \%-20 \%$ of sporadic CRCs (6), and some other types of cancers. The MSI phenotype in those cancers can result from inherited or spontaneous mutations in either $\mathrm{MLH1}$ or $\mathrm{MSH} 2$ or from epigenetic silencing of $M L H 1(7,8)$.

In mice, homozygous deletions of Msh2 $(9,10), M s h 3$ (11), or Msh6 (12) all lead to an increased incidence of tumors, including lymphomas, gastrointestinal (GI) cancers, and skin cancers. $\mathrm{Mlh1}^{-1}$ $(13,14)$ and $M l h 3^{-/-}(14)$ mice are also predisposed to lymphomas and GI tumors. There are some differences, however, among MMR

Conflict of interest: The authors have declared that no conflict of interest exists. Citation for this article: J. Clin. Invest. 119:2714-2724 (2009). doi:10.1172/JCI37405 homologs with respect to their involvement in tumor suppression. For example, $M s h 2^{---}$or $\mathrm{Mlh}^{-/-}$mice are the most cancer prone of these mice, with a median survival of only 6 months.

In contrast, CIN refers to loss or gain of either whole chromosomes or large fractions of a chromosome during cell division at an increased rate compared with normal cells. CIN leads to aneuploidy and an increased rate of loss of heterozygosity (LOH). Although CIN is the predominant form of genetic instability in most solid tumors, including CRCs, the molecular basis for CIN in cancer cells remains incompletely understood. Numerous observations suggest that error-free mitosis is essential for the maintenance of genome integrity. Mitotic checkpoints ensure normal progression through each phase of mitosis without errors. Early studies of CIN focused on the spindle assembly checkpoint, which inhibits sister chromatid separation until all chromosomes are properly attached to the mitotic spindles (15-17). Although the spindle assembly checkpoint is critical for the maintenance of chromosomal stability, mutations in known spindle assembly checkpoint components are rare in human cancers (18-24), suggesting that CIN might instead reflect disruption of other mitotic checkpoint pathways.

CHFR (checkpoint with forkhead and ring finger domains) is a relatively recently identified component of an early mitotic checkpoint that delays chromosome condensation and transition from prophase into metaphase in response to mitotic stress (25). The CHFR protein contains an $\mathrm{NH}_{2}$-terminal forkhead-associated (FHA) domain that is involved in phosphor-protein interactions (25), a central ring finger domain that participates in protein ubiquitination (26-28), and a C-terminal cysteine-rich region that is responsible for the interaction between CHFR and one of its target proteins, Aurora A (29). In addition to regulating the prophase to metaphase transition, possibly by regulating the appearance of kinases required for this transition (25), CHFR also appears to regulate chromosome segregation later in mitosis to maintain genomic 
Table 1

Patient and tumor characteristics

\begin{tabular}{lc} 
Characteristic & $\boldsymbol{n}(\%)$ \\
Age & \\
$<64$ & $17(34)$ \\
$\geq 64$ & $33(66)$ \\
Sex & \\
$\quad$ Male & $25(50)$ \\
Female & $25(50)$ \\
Tumor site & \\
Proximal & $37(74)$ \\
Distal & $13(26)$ \\
Duke stage & \\
B & $26(52)$ \\
C & $24(48)$ \\
Extent of the primary tumor & \\
T1, T2 & $2,7(4,14)$ \\
T3, T4 & $34,7(68,14)$ \\
Lymph node metastasis & \\
N0 & $26(52)$ \\
N1 & $13(26)$ \\
N2 & $11(22)$ \\
Distant lymph node metastasis & \\
M0 & $50(100)$ \\
M1 & $0(0)$ \\
MMR status & \\
MSS/MSI-L & \\
MSI-H & $40(80)$ \\
\hline
\end{tabular}

$n=50$ patients.

instability (29-32). Importantly, CHFR function is compromised in a variety of human tumors. This CHFR inactivation results from mutations in the CHFR gene or, more commonly, from epigenetic silencing of CHFR due to promoter hypermethylation or histone deacetylation (28, 33-38). The frequency of CHFR silencing raises the possibility that dysregulation of CHFR may contribute to CIN phenotype. Indeed, our previous studies have shown that targeted Chfr deletion leads to defective chromosomal segregation, aneuploidy, and increased tumor formation in mice (29).

It has long been speculated that cancers develop instability either at the nucleotide sequence level (MSI) or at the chromosome level (CIN) but not both. In the present study, however, gene expression profiling demonstrated low CHFR expression in the majority of MSI colon tumor samples examined. To further test the hypothesis that simultaneous inactivation of CHFR and MMR genes might synergistically promote tumorigenesis, we generated mice with targeted deletions of both Chfr and Mlh1. The resulting $\mathrm{Chfr}^{-/-} \mathrm{Mlh1}^{-/-}$ mice had decreased tumor latency and increased tumor incidence compared with their control littermates. Moreover, primary mouse embryonic fibroblasts (MEFs) from the Chfr-1- $M l h 1^{-1-}$ mice exhibited increased genomic instability. Collectively, these results not only raise the possibility that combined CIN and MIN can greatly enhance tumorigenesis, but also suggest that this combination might be responsible for the development of at least a subset of cancers in humans.

\section{Results}

In order to directly characterize the transcriptome specifically associated with microsatellite stable (MSS) and instable (MSI) colon cancer, respectively, we interrogated the gene expression profiling of these 2 subtypes of colon cancer with a microarray (Affymetrix GeneChip HGU133A) that contains 44,000 probe sets corresponding to approximately 33,000 human genes. Since a frequent epigenetic change in cancer involves aberrantly hypermethylated $\mathrm{CpG}$ islands in the promoter regions, with transcriptional silencing of these corresponding genes (reviewed in ref. 39), we also examined 1505 highly informative CPG sites spanning 807 genes using methylation array (Illumina) to identify hypermethylated CpG islands that are tumor specific. In this study, we included 50 primary colon cancers as well as 6 normal colorectal specimens. The clinical characteristics of the patients whose specimens were subjected to microarray analysis are summarized in Table 1.

Unexpectedly, we observed that the expression of CHFR was markedly suppressed in all colon cancer samples with high MSI (MSI-H) examined when compared with normal colon samples (Figure 1A). In contrast, 55\% of MSS/MSI-L (where MSI-L indicates low MSI) colon cancer samples displayed reduced CHFR expression (Figure 1B). Statistical analysis suggests that this specific downregulation of CHFR expression in MSI-H tumors is highly significant $(P<0.001)$. Analysis of gene expression data sets from an established cancer gene microarray meta-analysis public database (Oncomine Research; www.oncomine.org) independently demonstrated decreased CHFR expression in MSI-H tumors. Specifically, Koinuma et al. (40) investigated gene expression patterns in MSS as well as MSI CRC samples. Our analysis of this data set revealed that CHFR mRNA expression was significantly lower in MSI tumors than in MSS tumors $(P<0.001$, Student's $t$ test) (Figure 1C), which is in agreement with our findings. Notably, results from methylation array analyses indicated that the majority (87.5\%) of colon cancer samples with low CHFR expression were also hypermethylated in the CHFR promoter region (Figure 1B), suggesting that reduced expression of CHFR was primarily due to an epigenetic silencing.

Aurora A (also known as STK15/BTAK) is a serine/threonine kinase that plays a critical role in centrosome maturation and bipolar spindle assembly (41). Amplification and overexpression of Aurora A have been observed in many primary tumors including breast cancer, CRC, and gastric cancer (42-45). Like Chfr downregulation, Aurora A overexpression in WT MEFs induces defective chromosome segregation and cytokinesis failure, resulting in aneuploidy and polyploidy $(46,47)$. Phenotypic changes resulting from CHFR deficiency or Aurora A overexpression are very similar, suggesting they may regulate the same pathway or overlapping pathways. Indeed, our previous study demonstrated that Chfr ubiquitinates Aurora A and that Aurora A is upregulated in Chfrnull cells or mice (29).

To investigate the potential link between CHFR downregulation and Aurora A overexpression in human cancer, CHFR and Aurora A protein levels in colon specimens were examined by immunohistochemistry (IHC). Nuclear staining and diffuse cytoplasmic staining of Aurora A were evident (Figure 2, B, E, and H), consistent with previous studies (48-50). CHFR staining was localized primarily in the nucleus of the cells (Figure 2, A, D, and G). Whereas normal colon mucosa exhibited weak staining for Aurora A in crypt cells (Figure 2, B and C), strong nuclear CHFR staining was evident in normal apical colon mucosa cells (Figure 2, A and C). In cancer tissues, Aurora A staining exhibited an inverse correlation with CHFR (Spearman's rank correlation; correlation coefficient, $r=-0.53$ with $P=0.0001$ ) (Figure $2 \mathrm{~J}$ ), supporting the 

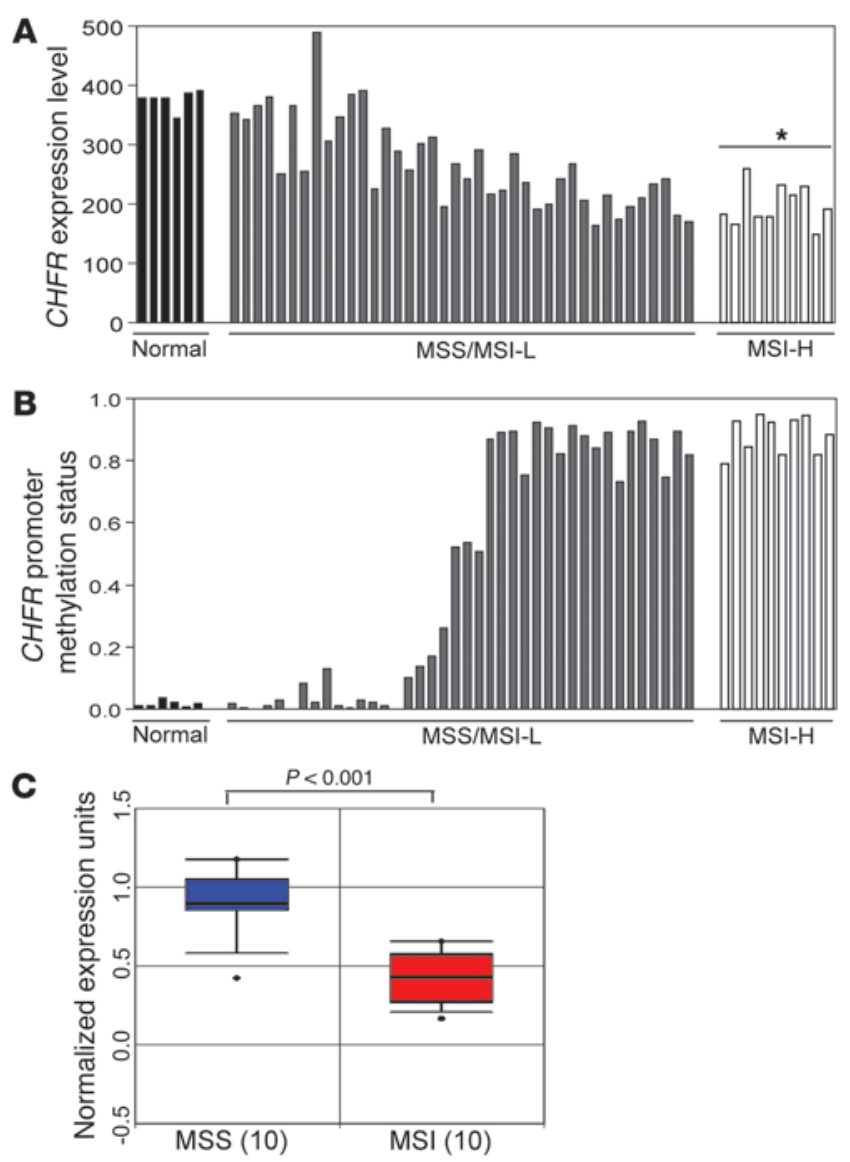

concept that CHFR downregulation may facilitate Aurora A overexpression and tumorigenesis.

Growing evidence has linked CHFR deficiency with CIN and tumorigenesis (29-31). The observation of loss of CHFR in all of the MSI-H samples prompted us to further explore the potential synergy between the CIN and MSI pathways in tumorigenesis. Specifically we determined whether deficiencies in both CHFR and MMR genes would synergistically increase tumor susceptibility. Accordingly, we generated mice deficient in both Chfr and Mlh1. Because mice with homozygous deletion of $M l h 1$ are sterile $(51,52)$, we bred recently described $\mathrm{Chfr}^{-/-}$mice (29) with $\mathrm{Mlh}^{+/-}$mice to generate $\mathrm{Chfr}{ }^{+/-} \mathrm{Mlh1^{+/ }}$ offspring. Double heterozygous mice were then interbred to generate cohorts of Chfr ${ }^{-1-} \mathrm{Mlh1}^{-/-}, \mathrm{Chfr}^{-/-} \mathrm{Mlh1}^{+/-}$, $\mathrm{Chfr}^{-/-} \mathrm{Mlh1}^{+/+}, \mathrm{Chfr}^{+/+} \mathrm{Mlh1}^{+/-}, \mathrm{Chfr}^{+/+} \mathrm{Mlh1}^{-/-}$, and $\mathrm{Chfr}^{+/+} \mathrm{Mlh}^{+/+}$mice for this study. All genotypes exhibited the expected Mendelian frequencies, indicating that Chfr deficiency does not affect early viability of Mlh1-deficient mice. Reduction or loss of Chfr and Mlh1 proteins was confirmed by Western blotting (Figure 3A).

Mice with the different Chfr/Mlh1 genotypes were monitored for survival. Consistent with previous findings $(13,14,29)$, both $\mathrm{Chfr}^{+/+}$ $M l h 1^{-/-}$and $C h f r^{-/} M l h 1^{+/+}$mice had shorter life spans than their WT littermates $(P<0.0001$ or $P<0.0001$, respectively) (Figure 3B). Fifty percent of $\mathrm{Chfr}^{-/-} \mathrm{Mlh}^{+/+}$mice died by the age of 18.6 months, whereas it took only 8.5 months for $50 \%$ of the $\mathrm{Chfr^{+/+ }} \mathrm{Mlh1}^{-/-}$mice to perish (Figure 3B), supporting our previous finding that Chfr functions as a tumor suppressor gene and that loss of Chfr results in a mild cancer predisposition phenotype. Importantly, the $\mathrm{Chfr}{ }^{-/-} \mathrm{Mlh}^{-1-}$ mice had a significant reduction in longevity (median survival 6.1

\section{Figure 1}

CHFR expression in colon cancer samples. (A) CHFR mRNA levels in 6 cases of normal colon mucosa, 40 cases of MSS/MSI-L colon cancer, and 10 cases of MSI-H colon cancer. ${ }^{*} P<0.001$, compared with normal colon samples and determined by Student's $t$ test. (B) Methylation status of CHFR promoter in those colon samples. Data are presented as the ratio of fluorescent signal from the methylated allele to the sum from the fluorescent signal from both methylated and unmethylated alleles. These values range from 0 (unmethylated) to 1 (methylated). (C) CHFR transcript levels in colon cancer measured using DNA microarray as reported by Koinuma et al. (40). There were 10 cases of MSS tumors and 10 cases of MSI tumors included in the study. $y$ axis represents normalized expression units. Box-and-whiskers plots indicate median values of the data and 25 th and 75 th percentiles of the data sets. Whiskers indicate minimum and maximum data values that are not outliers. Points outside of the ends are outliers. $P$ value was calculated by using the Student's $t$ test.

months) compared with other genotypes, including $\mathrm{Chfr}^{+/+} \mathrm{Mlb}^{-/-}$ littermates $(P=0.0002)$ (Figure 3B). Moreover, Chfr ${ }^{-/-} \mathrm{Mlh}^{+/-}$mice exhibited an intermediate phenotype with an average survival of 13.3 months, while $\mathrm{Chfr^{+/+ }} M l h 1^{+/-}$mice survived for an average of 22 months (Figure 3B).

Tumor incidence in these mice is summarized in Table 2. As expected, 9 of $30(30 \%)$ of $\mathrm{Chfr}^{+/+} \mathrm{Mlh1}^{+/-}$and 23 of $32(72 \%)$ of Chfr $\mathrm{fr}^{+/+}$ $M l b 1^{-/-}$mice developed tumors. The fact that the Chfr $\mathrm{fl}^{+/+} \mathrm{Mlh1^{+/- }}$ mice were moribund at an average age of 22 months, whereas the $\mathrm{Chfr} \mathrm{r}^{+/+} \mathrm{Mlh}^{-/-}$mice were moribund earlier (8.7 months), indicates that the $\mathrm{Chfr^{+/+ }} \mathrm{Mlb1}^{-/-}$mice develop tumors much faster than their heterozygous littermates. Importantly, 31 of 32 (97\%) mice deficient for both Chfr and Mlh1 (Chfr $\left.{ }^{-1} \mathrm{Mlh}^{-/-}\right)$developed tumors, with $16(50 \%)$ of those having multiple tumors. These mice became moribund with lymphomas as early as 2.8 months, and a majority succumbed to tumors by 10.5 months, with lymphomas and tumors of the GI tract and other organs (Figure 3C). A large portion (30\%) of these mice died with rapid onset, very aggressive $\mathrm{CD} 4^{+} \mathrm{CD} 8^{+} \mathrm{lym}$ phomas (Figure 3D). Lymphomas were found in multiple organs including lymph node, liver, spleen, lung, kidney, colon, and intestine (Figure 3C). This phenotype is similar to that observed in $M l h 1^{-/-}$mice $(13,14)$. Perhaps not surprisingly, Chfr ${ }^{-/-} M l b 1^{+/-}$mice developed tumors at an intermediate frequency (Table 2). These mice had a later onset of lymphoma and showed a relatively broad spectrum of tumors, including more mature B lineage lymphomas and other solid tumors. Based on this analysis of survival rate, tumor incidence, and tumor spectrum in cohorts of Chfr ${ }^{-1-} \mathrm{Mlh}^{-/-}$, $\mathrm{Chfr}^{-/-} \mathrm{Mlh1}^{+/-}, \mathrm{Chfr}^{-/-} \mathrm{Mlh1}^{+/+}, \mathrm{Chfr}^{+/+} \mathrm{Mlh1}^{+/-}, \mathrm{Chfr} \mathrm{r}^{+/+} \mathrm{Mlh1}^{-/-}$, and $\mathrm{Chfr} \mathrm{fr}^{+/+}$ $M l h 1^{+/+}$mice, we conclude that the $C h f r^{-/}$genotype substantially accelerates tumorigenesis in a Mlh1-deficient background.

We analyzed six lymphomas from $\mathrm{Chfr}^{+/+} \mathrm{Mlh1}^{+/-}$and $\mathrm{Chfr}^{+/+} \mathrm{Mlh1}^{-/-}$ mice, respectively, to determine whether any of them would exhibit Chfr downregulation, similar to that observed in human tumors. Interestingly, real-time RT-PCR as well as Western blotting analysis revealed that 1 out of $6 \mathrm{Chfr^{+/+ }} M \mathrm{Mh}^{-/-}$lymphomas showed a dramatic reduction in Chfr expression when compared with that in $\mathrm{Chfr} \mathrm{r}^{+/+}$ $M l b 1^{+/-}$tumors (Figure 3E), suggesting that Chfr loss occurred during tumorigenesis in Mlb1-deficient mice, similar to that observed in human tumors (Figure 1). We sequenced the promoter and exons of Chfr in the tumors, which showed decreased Chfr expression, but did not detect any mutations (data not shown; see Supplemental Figure 2 for the primers used; supplemental material available online with this article; doi:10.1172/JCI37405DS1). Thus, the loss 

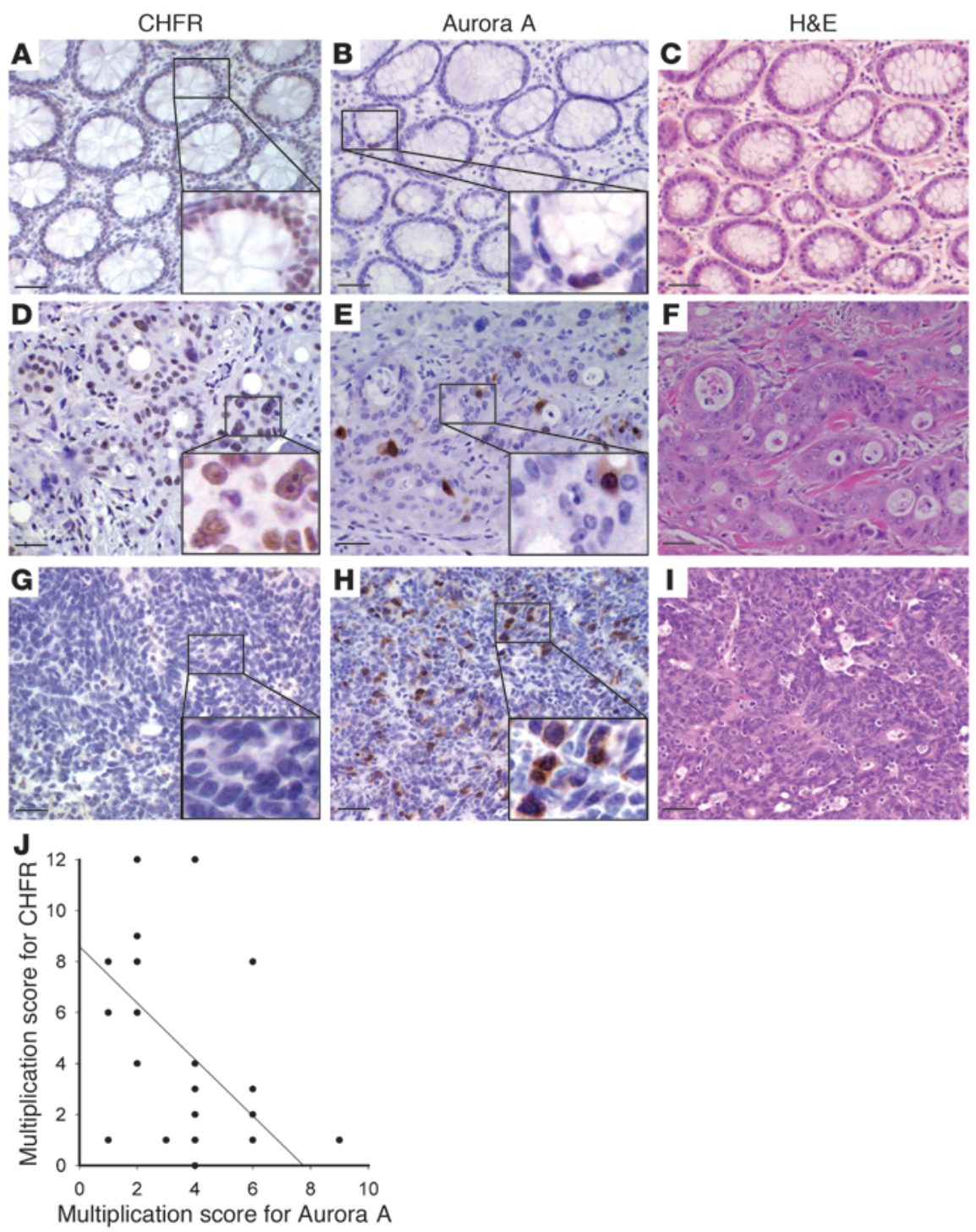

\section{Figure 2}

IHC analysis of CHFR and Aurora A protein levels in human colon specimens. Representative pictures of normal colon mucosa $(\mathbf{A}-\mathbf{C})$, MSS colon tumors (D-F), and MSI-H colon tumors (G-I) stained with anti-CHFR (A, D, and $\mathbf{G}$ ), anti-Aurora $\mathbf{A}(\mathbf{B}, \mathbf{E}$, and $\mathbf{H})$, and $\mathrm{H} \& \mathbf{E}$ (C, F, and I), respectively. Scale bars: $50 \mu \mathrm{M}$. (J) This scatter blot shows the inverse relationship between the IHC scores of Aurora A and CHFR staining in CRC tissue (Spearman's rank correlation, $r=-0.5315, P=0.0001)$. $\mathrm{IHC}$ scores include the percentage of positive cells and the intensity of the staining. Data points represent individual samples.

to the maintenance of chromosomal stability, as expected, Mlh1 has little or no effect on this process.

Next, we determined the average number of chromosomal aberrations per chromosome in these MEFs, which is an indicator of DNA repair deficiency. Approximately 0.0065 spontaneous chromosomal aberrations per chromosome were detected

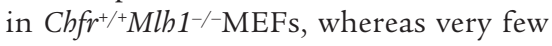
spontaneous chromosomal aberrations (0.0005/chromosome) were observed in WT MEFs (Figure 4C). Although Chfr-1$M l b 1^{+/+}$MEFs showed aneuploidy or polyploidy, fewer chromosomal aberrations $(0.002 /$ chromosome) were evident when compared with $\mathrm{Chfr}^{+/+} \mathrm{Mlb} 1^{-/-}$MEFs $(P<0.01$, ANOVA with Bonferroni's test).

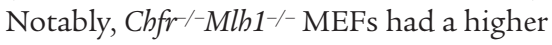
incidence of chromosomal aberrations (0.010/chromosome; $P<0.01$, compared with $\left.\mathrm{Chfr} \mathrm{r}^{+/+} \mathrm{Mlh1} 1^{-/-} \mathrm{MEFs}\right)$. Interestingly, severe spontaneous anomalies such as metacentric, fragmentation, and triradical

of Chfr expression in this tumor is likely to be caused by a mechanism other than missense mutation. Excitingly, the $\mathrm{Chfr^{+/+ }} M \mathrm{Mb}^{-/-}$ tumors with low Chfr expression displayed strong Aurora A expression, whereas the remaining $\mathrm{Chfr} \mathrm{r}^{++} \mathrm{Mlh1^{-/- }}$ or $\mathrm{Chfr} \mathrm{f}^{+/+} \mathrm{Mlh1^{+/- }}$ tumors had low or undetectable levels of Aurora A expression (Figure 3E). All $6 \mathrm{Chfr}^{-/-} \mathrm{Mlh}^{-/-}$lymphomas included as controls also exhibited enhanced Aurora A expression (Figure 3E), which agrees with observations in clinical colon cancers (Figure 2).

Since growing evidence suggests that Chfr plays an important role in maintaining chromosome stability, we next examined chromosomal stability in cells deficient for Chfr, Mlh1, or both. MEFs of all 6 genotypes were prepared from E13.5 embryos for in vitro culture. After 3 passages, metaphase spreads of MEFs were prepared and chromosome numbers were determined. About $45 \%$ of metaphase $C h \mathrm{fr}^{-/-} \mathrm{Mlh1^{-/- }}$ MEFs showed significant aneuploidy or polyploidy, which were similar to those of $\mathrm{Chfr}^{-1-} \mathrm{Mlh}^{+/-}$or $\mathrm{Chfr} \mathrm{f}^{-/}$ $\mathrm{Mlh1}^{+/+}$(37.7\% and 37.6\%, respectively) (Figure 4A). However, homozygous and heterozygous deletion of $\mathrm{Mlh} 1\left(\mathrm{Chfr}^{+/+} \mathrm{Mlh1}^{+/-}\right.$and $\left.\mathrm{Chfr^{+/+ }} \mathrm{Mlh1}^{-/-}\right)$exhibited nearly normal karyotypes, which is close to WT MEFs (Figure 4A), suggesting that while Chfr contributes configures were also observed in Chfr ${ }^{-1-} M l h 1^{-/-}$MEFs (Figure 4B). To further analyze DNA damage-induced chromosomal aberrations, we treated these MEFs with a low dose of ionizing radiation (IR) (1 Gy). WT MEFs efficiently repaired the majority of DNA breaks, with 0.017 chromosomal aberrations per chromosome (Figure 4D). In contrast, $\mathrm{Chfr^{+/+ }} \mathrm{Mlh1}^{-/-}$cells displayed more chromosomal aberrations than WT cells (Figure 4D) $(P<0.05$, ANOVA with Bonferroni's test). Chfr ${ }^{-1-} M l h 1^{-/-}$cells also showed defects in DNA damage repair, with 0.038 chromosomal aberrations per chromosome $(P<0.05$, compared with WT). These results suggest that DNA damage repair is attributed primarily to $M l h 1$ deficiency.

It has been reported previously that CHFR has an antiproliferative function, i.e., CHFR levels correlate inversely with mitotic index $(31,53)$. Therefore, we evaluated the mitotic index in Chfr/ Mlh1 mutant MEFs. All of the mutant MEFs $\left(\mathrm{Chfr}^{+/+} \mathrm{Mlh1}^{+/-}, \mathrm{Chfr^{+/+ }}\right.$ $\left.\mathrm{Mlb1}^{-/-}, \mathrm{Chfr}^{-/-} \mathrm{Mlb1}^{+/+}, \mathrm{Chfr}^{-/} \mathrm{Mlh1^{+/- }}, \mathrm{Chfr}^{-/-} \mathrm{Mlh1}^{-/-}\right)$exhibited a significant increase in mitotic cells (Figure 4E), as indicated by positive phospho-histone H3-Ser28 staining, when compared with WT MEFs ( $P<0.05$, as determined by ANOVA with Bonferroni's test). This agrees with our observations shown in Figure 3, B and C. 
A

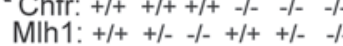
$\begin{array}{ll}m \text { Chfr } \\ --\infty & \text { Mlh1 } \\ -\infty & \text { B-Actin }\end{array}$

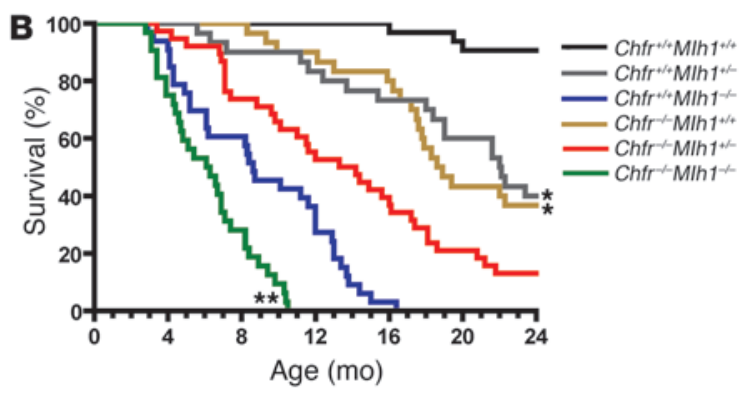

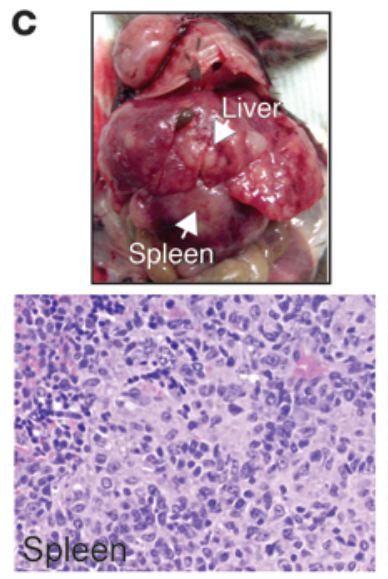

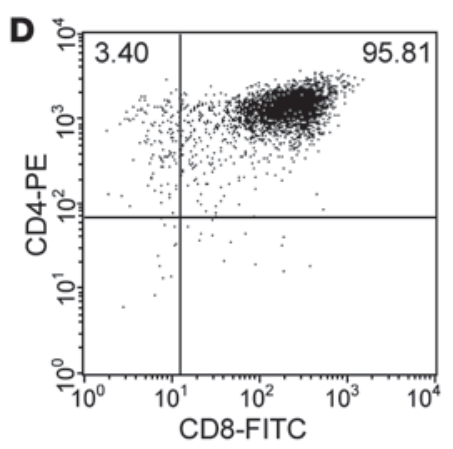

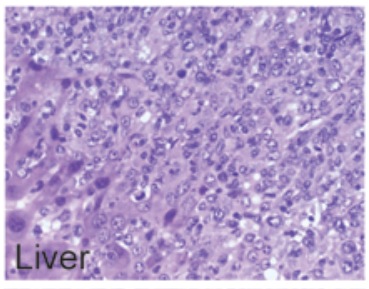

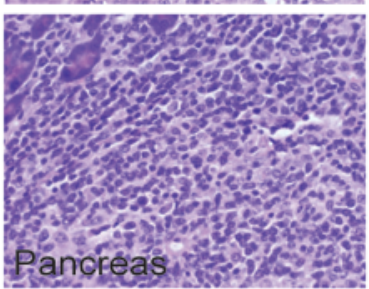

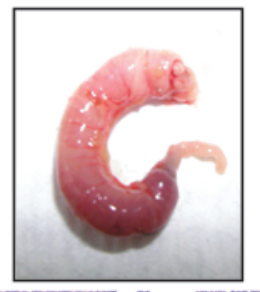

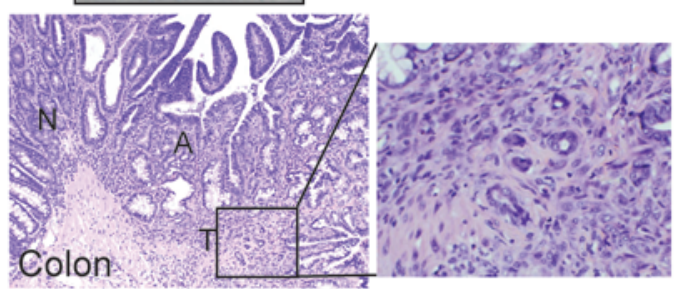

$\mathbf{E}$
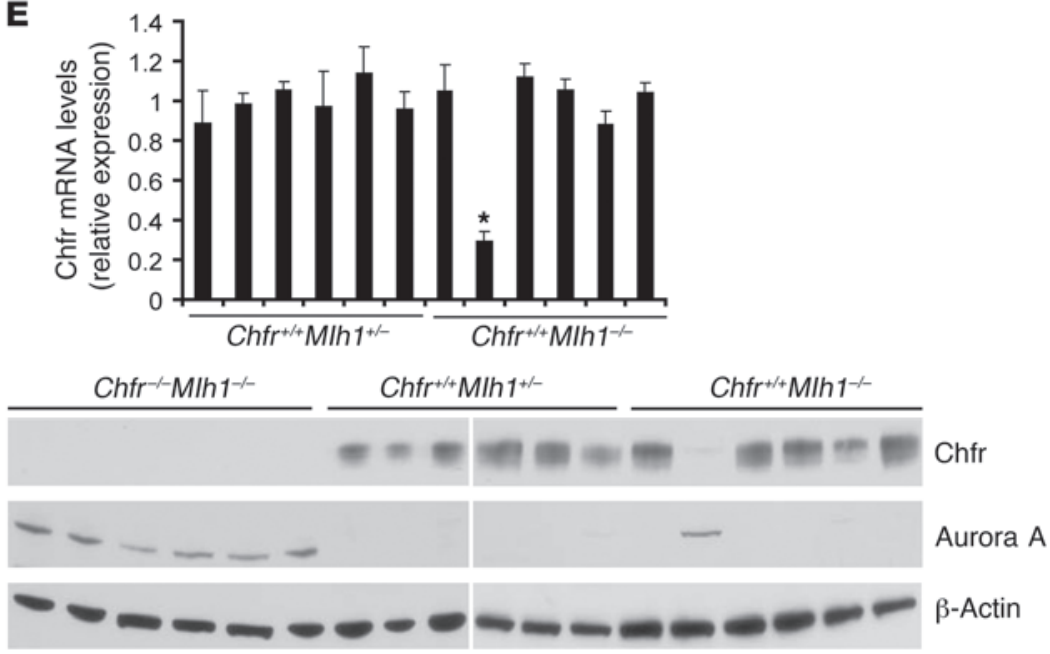

Figure 3

Deficiencies in both Chfr and Mlh1 increase spontaneous tumor incidence in mice. (A) Western blot of cell lysates from MEFs with indicated genotypes was performed using anti-Chfr and anti-Mlh1 antibodies, respectively. Anti- $\beta$-actin was used as a loading control. (B) Kaplan-Meier

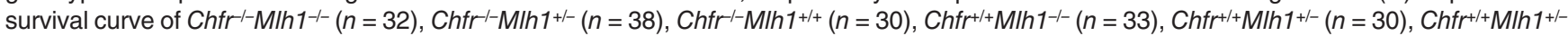
$(n=30)$, and $\mathrm{Chfr}^{+/+} \mathrm{Mlh1}^{+/+}(n=32)$ mice over a period of 24 months. ${ }^{*} P<0.0001$, compared with $C h f r^{+/+} \mathrm{Mlh} 1^{+/+}$mice; ${ }^{* *} P=0.0002$, compared

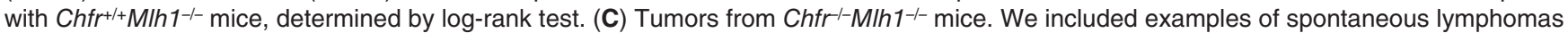
present in pancreas, liver, and spleen from the same $\mathrm{Chfr}^{-1-} \mathrm{Mlh1^{-1- }}$ mouse and a spontaneous colon cancer from another $\mathrm{Chfr}{ }^{-1-} \mathrm{Mlh}^{-1-}$ mouse as well as the H\&E-stained sections from those tumors. Original magnification, $\times 400$ (H\&E-stained sections from liver, spleen, and pancreas); $\times 100$ (left) and $\times 400$ (right) (H\&E-stained section from colon). N, normal; A, adenoma; T, adenocarcinoma. (D) CD4 and CD8 cell surface expression of cells from a representative spontaneous lymphoma was assessed by flow cytometry. Numbers in each quadrant indicate percentage of the total population. (E) Examination of Chfr expression in mouse lymphomas. RNA and protein from lymphomas with indicated genotypes were subjected to real-time RT-PCR (top panel) and Western blot (bottom panel), respectively. Thin lines in the bottom panel indicate that the samples were run on separate gels and the bands were spliced together at indicated positions. Results shown are mean \pm SD. Experiment was performed independently 3 times. ${ }^{*} P<0.001$, compared with $\mathrm{Chfr}^{+/+} \mathrm{Mlh} 1^{+/-}$tumors and determined by ANOVA. 
Table 2

Tumor incidence and tumor spectrum in Chfr/Mlh1 mutant mice

\begin{tabular}{|c|c|c|c|c|c|c|}
\hline Genotypes & Chfr $+/+M I h 1^{+/+}$ & Chfr+/+Mlh1+/- & Chfr+/+MIh1-/- & Chfr-/-MIh1+/+ & Chfr-/-MIh1+/- & $\mathrm{Chfr}^{-/-M I h 1^{-/-}}$ \\
\hline Mice in study & 32 & 30 & 32 & 30 & 38 & 32 \\
\hline Mice with tumors & 2 & 9 & 23 & 10 & 30 & 31 \\
\hline Mice with multiple tumors & 1 & 4 & 6 & 5 & 9 & 16 \\
\hline \multicolumn{7}{|l|}{ Tumor types } \\
\hline Lymphoma & 1 & 6 & 15 & 6 & 18 & 21 \\
\hline Gl tumor & & 3 & 8 & 2 & 9 & 12 \\
\hline Squamous cell & & & 1 & 1 & 1 & 2 \\
\hline Hepatocellular carcinoma & & & & & 1 & \\
\hline Spleen carcinoma & & & 1 & & & \\
\hline Mature teratoma & & & & 1 & & 1 \\
\hline Sarcoma & & & & & 1 & \\
\hline Lung adenocarcinoma & 1 & 1 & & & 1 & \\
\hline
\end{tabular}

These results suggest that both Chfr and Mlh1 have antiproliferative effects and that loss of their expression promotes cell proliferation and tumor growth.

\section{Discussion}

Several observations suggest a link between MSI and development of CIN. In the present study, microarray expression analysis revealed a frequent loss of CHFR expression in colon cancers with the MSI phenotype, implying a potential synergistic effect of these pathways during tumorigenesis. Consistent with our results, a recent report also suggested that inactivation of the $M L H 1$ gene is frequently accompanied by CHFR promoter hypermethylation in colon cancer (54). Furthermore, based on meta-analysis of the Oncomine database, we identified such a link in another study (Figure 1C) (40). More importantly, our in vivo data using Chfr-/$M l b 1^{-/-}$mice directly demonstrate that a deficiency of Chfr leads to a markedly increased predisposition to various tumors in the context of Mlh1 deficiency.

Although both CHFR and MLH1 contribute to genomic integrity, they function through different mechanisms, with CHFR deficiency triggering mild CIN and MLH1 deficiency mainly leading to MSI. Chfr ${ }^{-1-} M l h 1^{-/-}$mice developed very early onset of tumors compared with other genotypes, suggesting that both types of instability seem to occur early during tumorigenesis. Consistently, it has been reported that inactivation of CHFR or MLH1 is an early event in human carcinogenesis $(37,54-56)$. The molecular basis for predisposition to cancer in $\mathrm{Chfr}^{-/-} \mathrm{Mlh1^{-/- }}$ mice is still unclear, although, based on the known roles of each protein, it is likely that combined genetic instabilities would lead to accelerated activation or dysregulation of oncogenes and tumor suppressor genes and therefore result in rapid tumorigenesis.

In addition to a postreplicative MMR function, MMR proteins also act as sensors of DNA damage, triggering checkpoint activation and cell death responses $(57,58)$. For example, upon exposure to $\mathrm{S}_{\mathrm{N}} 1$ alkylating agents such as $N$-methyl- $N^{\prime}$-nitro- $N$-nitrosoguanidine (MNNG), MMR-proficient cells undergo $\mathrm{G}_{2} / \mathrm{M}$ cell cycle arrest followed by programmed cell death (59-61) (Supplemental Figure 4). The MMR system has also been implicated in S-phase checkpoint control in response to low doses of IR $(62,63)$. MMRdeficient CRC tumors and cell lines tend to accumulate mutations within microsatellite repeats of genes implicated in the double- strand break (DSB) repair pathway (e.g., MRE11 and RAD50) (64, 65). Furthermore, impaired MMR function results in inactivation of ATM and MRE11 genes, which correlates with impairment of the DSB repair system in leukemia and lymphoma cell lines (66). This may provide a plausible explanation for the increased frequency of chromosomal aberrations in Mlh1-null cells (Figure 4C). Therefore, although the increased mutation rate caused by an MMR defect may be sufficient to drive tumorigenesis, the DNA damageresponse function of Mlh1 may play an additional role that contributes to the synergistic tumorigenesis in Mlh1/Chfr double-knockout mice. Indeed, the frequency of spontaneous chromosomal aberrations significantly increased in $\mathrm{Mlh1}^{-/-}$or $\mathrm{Chfr} \mathrm{f}^{-/} \mathrm{Mlh}^{-/-}$cells (Figure 4C), suggesting that Mlh1 may directly or indirectly contribute to the suppression of chromosomal aberrations, including DSBs. In future studies, we would like to separate these 2 distinct functions of Mlh1 and to determine whether one or both are involved in the synergistic tumorigenesis observed in Chfr deficiency. In this regard, the demonstration that, for mice, carrying a homozygous $M l h 1^{\mathrm{G} 76 R}$ mutation, which disrupts the ATP processing ability of Mlh1 (67), leads to diminished DNA repair capacity without affecting the MMR-mediated DNA damage-response might provide a tool for addressing the relative contributions of these functions to tumor suppression in the face of Chfr loss.

CHFR is a recently identified mitotic checkpoint protein. The fact that CHFR is inactivated more frequently than all known spindle checkpoint genes combined $(18,19,22,68,69)$ and that CHFR expression is frequently lost in a variety of human cancers indicates its critical function in tumor suppression. This is fully supported by the demonstration that Chfr-null mice have an increased tumor incidence (ref. 29 and Figure 3). How CHFR suppresses tumor formation is not completely understood. Previous studies in cell lines demonstrated that Aurora A is a Chfr substrate and that Aurora A levels inversely correlate with Chfr (29). In the present study, we extended these findings to clinical colon cancer samples (Figure 2), providing clinical evidence that CHFR negatively regulates Aurora A. In view of the ability of Aurora A overexpression to induce a mild CIN phenotype, these observations provide at least one mechanism by which CHFR may participate in the control of genomic stability and tumor suppression. In Xenopus, Chfr has been shown to ubiquitinate Plk1 as well, leading to proteasomal Plk1 degradation (28). However, the role of CHFR in 


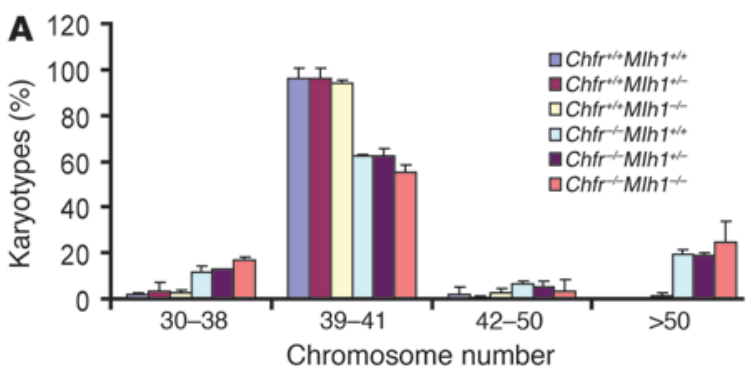

B
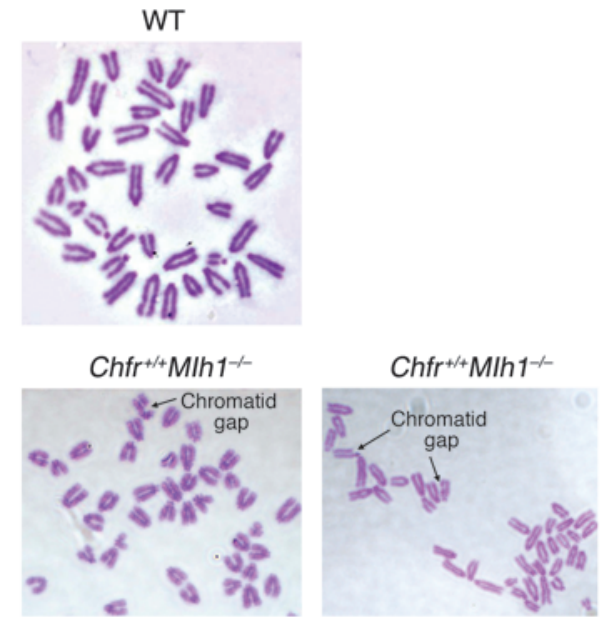

Chfr'--Mlh1-1-

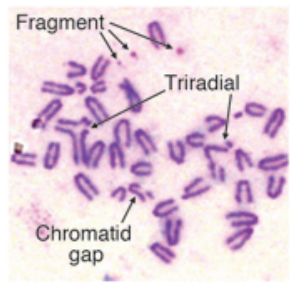

Chfr-l-MIh1 $^{-1-}$

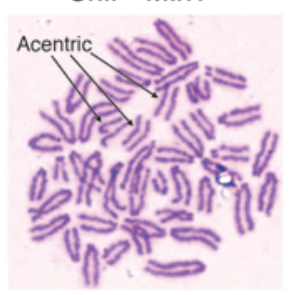

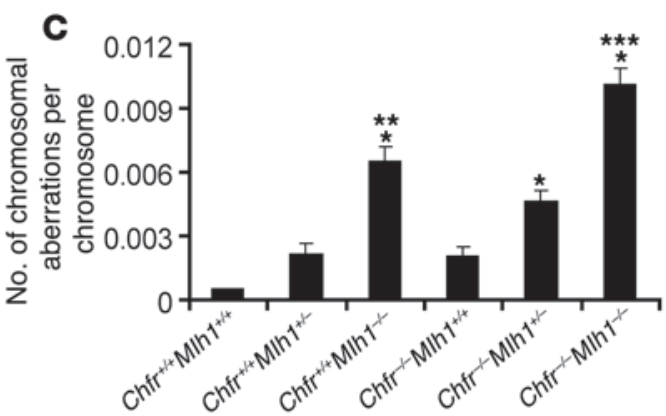

D
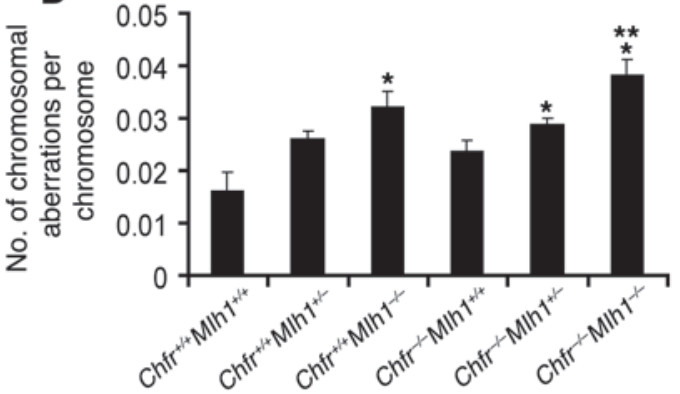

$\mathbf{E}$

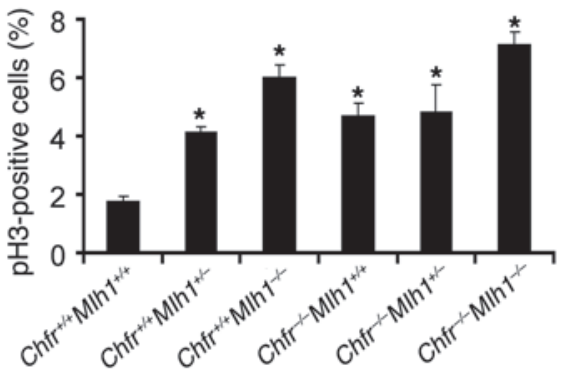

Figure 4

Deficiencies in both Chfr and Mlh1 lead to increased chromosomal aberrations. (A) Chfr-l-Mlh1-/- cells show a rate of aneuploidy similar to that of

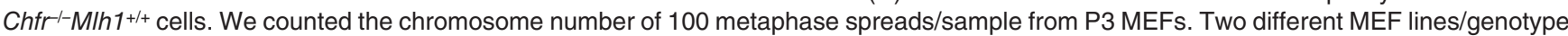
were used. (B) Representative pictures of metaphase spreads from MEFs (WT, Chfr ${ }^{-1} \mathrm{Mlh1} 1^{-/}$, and $\mathrm{Chfr}{ }^{+/+} \mathrm{Mlh} 1^{-/-}$). Arrows indicate different types of aberrations. Original magnification, $\times 1000$. (C) Spontaneous chromosomal aberrations were evaluated in MEFs with indicated genotypes. ${ }^{*} P<0.01$,

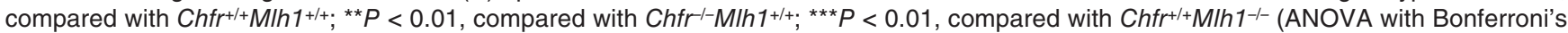
test). (D) DNA damage-induced chromosomal aberrations were scored in MEFs with indicated genotypes. MEFs were treated with 1 Gy of IR

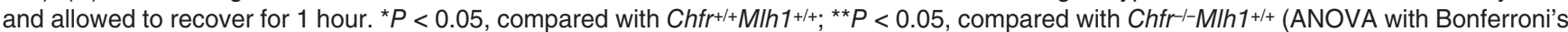
test). Two different MEF lines/genotype were used in C and D. Columns indicate mean values from 2 MEF lines; error bars show SD. Number of chromosomal aberrations per chromosome is presented. Results of individual MEF line from $\mathbf{C}$ and $\mathbf{D}$ are presented in Supplemental Figure 3. (E) Mitotic index was determined in MEFs with indicated genotypes. Two different MEF lines/genotype were used. The mitotic index is presented as the average percentage of histone H3-Ser28-stained nuclei. Over 1000 DAPI-stained nuclei were evaluated for each genotype from triplicate experiments. ${ }^{*} P<0.05$, compared with $\mathrm{Chfr}^{+++} \mathrm{Mlh1^{+/+ }}$ (ANOVA with Bonferroni's test). Results shown are mean \pm SD.

regulating Plk1 activity or protein levels in mammalian cells has been controversial (70-72) and remains to be further explored. In addition, CHFR also functions as a negative regulator of proliferation as well as cellular mobility and invasion in cell culture models $(31,53,73)$. All of these activities could also contribute to the role of CHFR in tumor suppression.

To date, there are no definitive studies showing a correlation between CHFR and aneuploidy (or CIN) in human cancers. This may reflect the mild degree of CIN associated with CHFR deficiency and the difficulty in accurately assessing CIN in clinical specimens. In the clinical setting, CIN is routinely judged by karyotypic studies, which only show the gain or loss of whole or large portions of chromosomes. Molecular studies indicate that karyotypic results usually underestimate the frequency of aneuploidy (74). In addition, there are few assays currently available in the clinic setting that can reliably measure other chromosomal aberrations such as rearrangements, deletions, insertions, inversions, and amplifications. These changes occur frequently, which in theory should be considered as another characteristic hallmark of the CIN phenotype $(75,76)$. Consistently, MEFs from 
Chfr $/-M l h 1^{-/-}$mice exhibit an increased incidence of chromosomal aberrations. Therefore, it is tempting to speculate that loss of CHFR may contribute to subtle chromosomal aberrations as well as gross changes and that 2 distinct mechanisms (CIN and MSI) may converge and lead to the development of at least a subset of human cancers. Prospective studies of the frequency of CHFR inactivation as well as careful evaluation of chromosomal aberrations in MSI tumors are needed to further assess the convergence of these 2 mechanisms.

The synergistic effects of Chfr loss and Mlb1 inactivation observed in this study are reminiscent of changes observed in $A T R^{+/-} \mathrm{Mlh1}^{-/-}$ mice (77). The ataxia-telangiectasia mutated (ATM) and ATM and RAD3-related (ATR) protein kinases act as master regulators of the DNA damage response by signaling to control cell-cycle transitions, DNA replication, DNA repair, and apoptosis (reviewed in ref. 78). While the ATM pathway responds to DNA DSBs, the ATR pathway responds primarily to agents that interfere with normal DNA replication. ATR is involved in the maintenance of DNA replication fidelity, and disruptions of the ATR pathway result in genomic instability. Mice bearing the $A T R^{+/-} M l h 1^{-/-}$genotype exhibit early tumor development. By 9 months of age, $71 \%$ of $A T R^{+/-} \mathrm{Mlh1}^{-/-}$mice develop cancer, particularly lymphomas and GI tumors, a spectrum similar to that observed in $\mathrm{Chfr}^{-1-} \mathrm{Mlh}^{-/-}$ mice. Further, $A T R^{+/-} M l b 1^{-/-}$MEFs, like Chfr $/-M l h 1^{-/-}$MEFs, exhibit substantially increased genetic instability. However, there are also some differences between these 2 models. While $A T R^{+/-}$ $M l h 1^{-/-}$mice are prone to embryonic lethality, $\mathrm{Chfr} /-\mathrm{Mlh}^{-/-}$mice showed no such defects, suggesting that loss of both Chfr and Mlh1 has no adverse effect on embryonic development, but specifically promoted tumorigenesis. This may be the reason that $\mathrm{Chfr} / \mathrm{MLH}$ double deficiency is more common in human cancers.

While additional studies are required to understand the cooperative nature of MSI and CHFR downregulation during tumorigenesis, the present finding of aberrant CHFR methylation in a large percentage of colon tumors, especially in MSI colon tumors, suggests a potential therapeutic strategy as well. Our previous studies and the studies of others (79-82) demonstrated that MSI tumors respond poorly to 5-fluorouracil (5-FU) therapy, and alternative chemotherapeutic regimens for patients with MSI tumors are needed to improve overall survival. Interestingly, cancer cell lines with low CHFR expression exhibit heightened susceptibility to the microtubule destabilizers $(25,27)$. Consistently, we analyzed the response of Chfr/Mlh1-deficient cells toward treatment with an antimicrotubule drug as well as DNA damage-inducing agents. As shown in Supplemental Figure 4, deficiency in Chfr, but not in Mlh1, sensitizes cells to nocodazole treatment. These findings would raise the possibility that suitable microtubule inhibitors might be a promising alternative for patients with MSI tumors that have low CHFR expression. In other words, CHFR expression status may serve as a useful molecular biomarker for predicting the sensitivity to particular chemotherapeutic agents in patients with MSI tumors. Further clinical studies are required to test this hypothesis.

In summary, we observed that loss of CHFR expression is frequent in MSI-H colon tumors. Further analysis demonstrated that simultaneous loss of Chfr and Mlh1 synergistically increased predisposition to cancer development in a murine model. These findings provide a plausible mechanism by which genetic instability contributes to tumorigenesis and suggest a possible therapeutic strategy for MSI tumors.

\section{Methods}

Profiled patient population. Tumors were selected from a prospective group of patients who had colon or rectal resections performed at the Mayo Clinic from 1995 to 1998, which has been previously described (83). Eligibility criteria for the current study included age at surgery greater than 50 (mean age 70), stage (Dukes B or C), known MMR status as determined by IHC and/or microsatellite testing, and colon tumors only (rectals excluded). Based on MMR status, those patients were divided into 2 groups: MSS/ MSI-L and MSI-H. All tissue procurement was approved by the Mayo Clinic Institutional Review Board.

$R N A$ isolation and sample preparation/scanning for Affymetrix GeneChip expression profiling. Frozen tumor tissue was cut, $\mathrm{H} \& \mathrm{E}$ stained, and reviewed by a GI pathologist. Areas containing no less than $70 \%$ tumor were macrodissected and confirmed by H\&E. Tissue was processed into tubes, ending with an additional H\&E slide to confirm that tumor tissue had not been depleted or shifted during sectioning. Total RNA was isolated from colonic tumor cells that were homogenized in $1 \mathrm{ml}$ of TRIzOL Reagent (Life Technologies); RNA was extracted according to the manufacturer's protocol and purified using commercial affinity resin column kits (QIAGEN)

The quality of the RNA was evaluated by obtaining electropherograms on Agilent 2100 Bioanalyzer and RNA integrity number (RIN) using 2100 Expert software (Agilent Technologies). RNA was processed and hybridized to the Affymetrix chip (HU133A; Affymetrix) according to the manufacturer's instructions.

GeneChip data analysis. GCOS 1.3 (Affymetrix) was used to scan and quantitatively analyze the scanned image. The perfect match probe level data were normalized using fastlo (84), a nonlinear normalization algorithm similar to cyclic loess. Data from all arrays were normalized. Linear mixedeffect models (85) were used to assess significance of comparisons between groups. The models included effects for tumor site, tumor stage, and sex due to the reported differences in gene expression by tumor site $(86,87)$ and stage (88) along with reported hormonal influences in colon cancer (89). Genes were ranked according to $P$ values, a measure of the change in expression relative to the variability in the data. All analyses were performed on the log base 2 scale.

Illumina methylation assay. For the methylation assay (Illumina), bisulfite-modified DNAs (250 ng) were first biotinylated. A methylation assay pool (OMA) containing query oligos for the CpG sites was allowed to hybridize, extend, and ligate to create a template for PCR. Following a universal PCR using fluorescently labeled primers, the DNA was hybridized to the BeadArray (Sentrix Array Matrix or BeadChips) and scanned. Analysis of methylation data took place in BeadStudio software, using the methylation software module. The methylation status of the target CPG sites was determined by comparing the ratio of fluorescent signal from the methylated allele to the sum from the fluorescent signal from both methylated and unmethylated alleles. These values range from 0 (unmethylated) to 1 (methylated).

Antibody. Mouse monoclonal anti-CHFR antibodies were developed in our laboratory and raised against full-length CHFR. The specificities of the CHFR antibodies were confirmed by Western blot analysis using cell lysates prepared from T47D cells transduced with either CHFR retroviral shRNA (RHS1764-9494026; Open Biosystems) or control vector (Supplemental Figure 1B). Decrease of signal on Western blots with T47D cells transduced with CHFR shRNA, but not the control vector, indicated that these antibodies specifically recognize endogenous CHFR protein. Further validation of the specificity of these antibodies was provided by IHC analysis of HeLa (absence of CHFR expression) and HeLa cells that ectopically express HA-tagged CHFR (Supplemental Figure 1A). The lack of signal on IHC with HeLa cells, but not the HeLa cells ectopically expressing CHFR, confirmed the specificity of these antibodies. Monoclonal anti-Aurora A anti- 
bodies used for IHC were generated in our laboratory and raised against full-length Aurora A (90). Anti-Mlh1 antibody was purchase from Calbiochem. Anti-HSP90 antibody was a gift from David Toft (Mayo Clinic).

IHC of human tissue. Standard avidin-biotin complex IHC was used. In brief, antigen retrieval was performed by steaming the slides for 30 minutes in $1 \mathrm{mM}$ EDTA buffer at $\mathrm{pH} 9.0$ in a steamer. The slides were then incubated sequentially with primary antibody, biotinylated secondary antibody, avidin-biotin complex, and chromogenic substrate 3, 30-diaminobenzidine (DAKO). CHFR and Aurora A protein expression was detected using a monoclonal anti-CHFR (1:1000 dilution) and anti-Aurora A antibody (1:50 dilution), respectively. Primary antibody was replaced with an equal concentration of nonimmune mouse IgG on control sections.

The scoring of Aurora A and CHFR staining (both cytoplasmic and nuclear) was conducted by 2 pathologists (L. Zhang and M.H. Muders), who evaluated 5 to 10 high-power fields ( $\times 40$ magnification) independently in a blinded fashion. Correlation of scoring between the 2 pathologists was significant $(P<0.0001$ with $r=0.7933)$. In the case of discrepancies in the scoring, the slides were discussed and cases were reviewed together to reach an agreement. The scoring of Aurora A was performed, with Aurora A intensity scored as follows: 0, negative; 1 , weak; 2, intermediate; and 3, strong. Quantity was scored in a semiquantitative manner. Staining of $1 \%-10 \%$ of cells was scored as 1 ; staining of more than $11 \%$ and less than $20 \%$ of cells was scored as 2 ; and staining of more than $20 \%$ of cells was scored as 3 . These cutoffs represent the first and third quartile. The median of the quantity of cells positive for Aurora A was $15 \%$, the first quartile was $12 \%$, and the third quartile was $20 \%$. In no cases did more than $35 \%$ of the cells stain positive for Aurora A. A multiplication score was created (total scores from 0-9, with 0 meaning no staining at all). A similar scoring system was used for CHFR. Intensity was scored from 0-3. Quantity was scored as 1 when $1 \%-25 \%$ of cells were stained, as 2 when $26 \%-50 \%$ of cells were stained, as 3 when $51 \%-75 \%$ of cells were stained, and as 4 when more than $75 \%$ of cells were stained. The statistical evaluation was performed using Spearman's rank correlations of the ordinal score data (multiplication scores). $P<0.05$ was considered statistically significant. Rho $(r)$ data represent the magnitude of the correlation $(-1$ to +1$)$. Results are displayed as a scatter blot. Statistical analysis was performed by M.H. Muders.

Generation of Chfr/Mlb1-double-knockout mice, genotyping, and MEFs. Mlh1 ${ }^{+/-}$ female mice (a gift from Marilia Cascalho, Mayo Clinic) were crossed with Chfr /- male mice (29) to generate $\mathrm{Chfr} \mathrm{r}^{+/} \mathrm{Mlh1^{+/- }}$ offspring. Both $\mathrm{Mlh1^{+- }}$ and $\mathrm{Chfr}^{-/}$mice were generated in a 129/C57BL/6 mixed background. To minimize the contribution of different genetic compositions of mouse strains and to allow for rigorous analysis of the tumor incidence, only littermates from $\mathrm{Chfr}^{+/-} \mathrm{Mlh1}^{+/-}$intercrosses were included. Mice were observed daily for signs of poor health, and moribund mice were sacrificed and examined for tumors throughout the body. Experimental procedures involving the use of these laboratory mice were reviewed and approved by the Institutional Animal Care and Use Committee of the Mayo Clinic.

Animals were genotyped by PCR of tail DNA. For Mlh1 genotyping, the following primers were used: primer A, TGTCAATAGGCTGCCCTAGG; primer B, TGGAAGGATTGGAGCTGCTG; and primer C, TTTTCAGTGCAGCCTATGCTC. The reaction was performed in a $25-\mu 1$ reaction mixture containing $50 \mathrm{ng}$ DNA, $5 \mathrm{ng} / \mu \mathrm{l}$ primer A and $5 \mathrm{ng} / \mu \mathrm{l}$ of primers $\mathrm{B}$ and $\mathrm{C}, 0.6 \mathrm{mM} \mathrm{MgCl}_{2}, 0.1 \mathrm{mM}$ each dNTP, and 0.25 units of Taq polymerase. Cycling conditions were 5 minutes at $91.5^{\circ} \mathrm{C}$ ( 1 cycle); 1 minute at $91.5^{\circ} \mathrm{C}, 1$ minute at $58^{\circ} \mathrm{C}$, and 30 seconds at $72^{\circ} \mathrm{C}(35$ cycles $)$; and 5 minutes at $72^{\circ} \mathrm{C}(1 \mathrm{cycle})$. The presence of the WT allele is indicated by a 350-bp PCR fragment (primers A and C) and the mutant allele by a 450-bp PCR fragment (primers A and B). For Chfr genotyping, the following primers were used: primer A, CTGTTATCTGTTGAGCCGTTTGTGAG; primer B, AAGTTTACAGTGATCTCCAGAGACCAG; and primer C, TGGATGTG-
GAATGTGTGCGAGG. The reaction was performed in a $25-\mu \mathrm{l}$ reaction mixture containing $50 \mathrm{ng}$ DNA, $5 \mathrm{ng} / \mu \mathrm{l}$ primer A and $5 \mathrm{ng} / \mu \mathrm{l}$ of primers $\mathrm{B}$ and $\mathrm{C}, 0.6 \mathrm{mM} \mathrm{MgCl}_{2}, 0.1 \mathrm{mM}$ each $\mathrm{dNTP}$, and 0.25 units of Taq polymerase. Cycling conditions were 5 minutes at $91.5^{\circ} \mathrm{C}$ ( 1 cycle); 1 minute at $91.5^{\circ} \mathrm{C}, 1$ minute at $60^{\circ} \mathrm{C}$, and 30 seconds at $72^{\circ} \mathrm{C}(35$ cycles $)$; and 5 minutes at $72^{\circ} \mathrm{C}(1 \mathrm{cycle})$. The presence of the WT allele is indicated by a 400-bp PCR fragment (primers A and B) and the mutant allele by a 400-bp PCR fragment (primers A and C). All MEFs were generated from E13.5 to E14.5 embryos using standard procedures (29).

Metaphase spreads. Two MEF lines of each genotype were used. Passage 3 MEFs were incubated with $50 \mathrm{ng} \mathrm{ml}^{-1}$ colcemid for 3-4 hours. Cells were collected, washed with PBS, resuspended in $75 \mathrm{mM}$ potassium chloride (KCL), and incubated at room temperature for 15 minutes. Cells were fixed in Carnoy's solution (75\% methanol and $25 \%$ acetic acid), and $15-\mu 1$ aliquots were dropped onto slides and stained with 55 Giemsa solution. Metaphase spreads were observed using a microscope (Eclipse 80i; Nikon) with a $\times 100$ NA 1.3 oil objective lens at room temperature. Spreads were photographed and analyzed using a camera (Spot 2 Megasample; Diagnostic Instruments Inc.) and Spot software 4.6 (Diagnostic Instruments Inc.).

For the IR-treated group, MEFs were irradiated (1 Gy) and allowed to recover for 1 hour before the addition of colcemid. Metaphase spreads were prepared as described above.

Real-time PCR. Total RNA was extracted from mouse tumors using Trizol (Invitrogen) according to the manufacturer's instructions. A 2-step real-time PCR was performed using cDNA prepared from RNA using a Superscript III First-Strand cDNA Synthesis Kit (Invitrogen) and a SYBR Green PCR Master Mix (Applied Biosystems) on an ABI PRISM 7700 SDS instrument following the manufacturer's instructions (91). The fold change in expression levels (using GAPDH as internal control) was determined by a comparative $\mathrm{Ct}$ method using the formula $2^{-\Delta \Delta \mathrm{Ct}}$, where $\mathrm{Ct}$ is the threshold cycle of amplification. GAPDH control primers were purchased from Applied Biosystems. For Chfr, the following primers were used: forward primer, TGCAAAAACCACATCCTGAA; reverse primer, TGGGTTGCAGCATATCTTGA.

Western blotting. Cell lysates were prepared from MEFs, and Western blots were performed using anti-Chfr and anti-Mlh1 antibodies. Cell lysates prepared from mouse tumors were subjected to immunoblotting using anti-Chfr and anti-Aurora A antibodies. Anti- $\beta$-actin was used for loading controls.

Characterization of tumors. Tissues from solid tumors were analyzed by standard histological analysis. Lymphomas were characterized by flow cytometry using anti-CD3, anti-CD4, anti-CD8, anti-CD19, anti-B220, anti-Thy1.2, and anti-IgM antibodies (BD Biosciences - Pharmingen).

Mutation screening. Sequencing of the promoter and exonic regions of the mouse Chfr gene (NM_172717) was performed on mouse tumor samples using dye termination chemistry (Big Dye Terminator with the model 3730xl sequencer; Applied Biosystems). Mouse Chfr gene contains 18 exons spanning approximately $37 \mathrm{~kb}$ on chromosome 5:110563879-110600988. We used 21 sets of primers to amplify all 18 exons and approximately $1 \mathrm{~kb}$ of the promoter region. Primer sets for PCR were designed using the webbased design tool Primer 3 (see Supplemental Figure 2). PCR was carried out using AmpliTaq Gold DNA Polymerase (Applied Biosystems) based on the standard protocol. After PCR reactions, the amplicons were treated with Exo-SAP (USB Corp.) to degrade unincorporated PCR primers and deoxynucleotide triphosphates. The cleaned products were mixed with the forward or reverse PCR primers for Sanger sequencing. The sequence variants were screened using Mutation Surveyor 2.41 (SoftGenetics).

Soluble tetrazolium salt assay. Cells were plated in 96-well tissue culture plates at a density of $5 \times 10^{3}$ cells per well. The next day, cells were treated with vehicle (DMSO) or increasing concentrations of either MNNG (Sigma-Aldrich) or nocodazole (Sigma-Aldrich), and cell viability was 
assessed after 72 hours by means of a CellTiter 96 Aqueous One solution cell proliferation assay (Promega) according to the manufacturer's instructions. Experiments were performed in triplicate.

Measure of apoptosis. To assess the extent of apoptosis after drug treatment, cells were treated with vehicle (DMSO) or increasing concentration of either MNNG or nocodazole. After 72 hours, the entire cell population was subjected to double staining for FITC-annexin V and PI using FITC-annexin V apoptosis detection kit (BD Biosciences) and analyzed by flow cytometry for apoptotic events according to the manufacturer's instructions.

Statistics. Survival curves and statistical analyses were performed using GraphPad Prism 4.0 software and the SAS software package (SAS). Correlation of CHFR and Aurora A protein expression was determined using Spearman's rank analysis. For all tests, the level of significance was set at $P<0.05$.

\section{Acknowledgments}

We are grateful to the Mayo Cytogenetic facility for performing the karyotype analysis and the Mayo Monoclonal Core facility for help with antibody production. This work was supported in part by grants from the NIH (CA125747 and CA121277 to D.J. Tindall; CA113381 to J. Chen), Mayo SPORE P50 (CA116201 project no. 1 to J. Chen and S.H. Kaufmann), and the T.J. Martell Foundation (to D.J. Tindall). J. Chen is a recipient of an Era of Hope Scholars award from the Department of Defense. Z. Fu is a recipient of a Ruth L. Kirschstein NRSA individual Fellowship (F32) from the NIH.

Received for publication September 10, 2008, and accepted in revised form June 24, 2009.

Address correspondence to: Donald James Tindall, GU 1742C, 200 First Street SW, Rochester, MN 55905, USA. Phone: (507) 2848139; Fax: (507) 284-2384; E-mail: tindall.donald@mayo.edu. Or to: Junjie Chen, The University of Texas MD Anderson Cancer Center, 1515 Holcombe Boulevard - Unit 66 (Room Y3.6006), Houston, TX 77030, USA. Phone: (713) 792-4863; Fax: (713) 794-5369; E-mail: jchen8@mdanderson.org. Or to: Zheng Fu, GU1742B, 200 First Street SW, Rochester, MN 55905, USA. Phone: (507) 2848138; Fax: (507) 284-2384; E-mail: fu.zheng@mayo.edu.

Junjie Chen's present address is: The University of Texas MD Anderson Cancer Center, Houston, Texas, USA.
1. Lengauer, C., Kinzler, K.W., and Vogelstein, B. 1998. Genetic instabilities in human cancers. Nature. 396:643-649.

2. Parsons, R., et al. 1993. Hypermutability and mismatch repair deficiency in RER+ tumor cells. Cell. 75:1227-1236

3. Bhattacharyya, N.P., Skandalis, A., Ganesh, A., Groden, J., and Meuth, M. 1994. Mutator phenotypes in human colorectal carcinoma cell lines. Proc. Natl. Acad. Sci. U. S. A. 91:6319-6323.

4. Eshleman, J.R., et al. 1995. Increased mutation rate at the hprt locus accompanies microsatellite instability in colon cancer. Oncogene. 10:33-37.

5. Aaltonen, L.A., et al. 1993. Clues to the pathogenesis of familial colorectal cancer. Science. 260:812-816.

6. Thibodeau, S.N., Bren, G., and Schaid, D. 1993. Microsatellite instability in cancer of the proximal colon. Science. 260:816-819.

7. Herman, J.G., et al. 1998. Incidence and functional consequences of hMLH1 promoter hypermethylation in colorectal carcinoma. Proc. Natl. Acad. Sci. U. S. A. 95:6870-6875.

8. Papadopoulos, N., and Lindblom, A. 1997. Molecular basis of HNPCC: mutations of MMR genes. Hum. Mutat. 10:89-99.

9. Reitmair, A.H., et al. 1995. MSH2 deficient mice are viable and susceptible to lymphoid tumours. Nat. Genet. 11:64-70.

10. de Wind, N., Dekker, M., Berns, A., Radman, M., and te Riele, H. 1995. Inactivation of the mouse Msh2 gene results in mismatch repair deficiency, methylation tolerance, hyperrecombination, and predisposition to cancer. Cell. 82:321-330.

11. Edelmann, W., et al. 2000. The DNA mismatch repair genes Msh3 and Msh6 cooperate in intestinal tumor suppression. Cancer Res. 60:803-807.

12. Edelmann, W., et al. 1997. Mutation in the mismatch repair gene Msh6 causes cancer susceptibility. Cell. 91:467-477.

13. Prolla, T.A., et al. 1998. Tumour susceptibility and spontaneous mutation in mice deficient in Mlh1, Pms1 and Pms2 DNA mismatch repair. Nat. Genet. 18:276-279.

14. Chen, P.C., et al. 2005. Contributions by MutL homologues Mlh3 and Pms2 to DNA mismatch repair and tumor suppression in the mouse. Cancer Res. 65:8662-8670.

15. Wassmann, K., and Benezra, R. 2001. Mitotic checkpoints: from yeast to cancer. Curr. Opin. Genet. Dev. 11:83-90.

16. Shah, J.V., and Cleveland, D.W. 2000. Waiting for anaphase: Mad2 and the spindle assembly checkpoint. Cell. 103:997-1000.

17. Musacchio, A., and Hardwick, K.G. 2002. The spindle checkpoint: structural insights into dynamic signalling. Nat. Rev. Mol. Cell Biol. 3:731-741.

18. Cahill, D.P., et al. 1999. Characterization of MAD2B and other mitotic spindle checkpoint genes. Genomics. 58:181-187.

19. Cahill, D.P., et al. 1998. Mutations of mitotic checkpoint genes in human cancers. Nature. 392:300-303.

20. Imai, Y., Shiratori, Y., Kato, N., Inoue, T., and Omata, M. 1999. Mutational inactivation of mitotic checkpoint genes, hsMAD2 and hBUB1, is rare in sporadic digestive tract cancers. Jpn. J. Cancer Res. 90:837-840.

21. Sato, M., et al. 2000. Infrequent mutation of the hBUB1 and hBUBR1 genes in human lung cancer. Jpn. J. Cancer Res. 91:504-509.

22. Yamaguchi, K., et al. 1999. Mutation analysis of hBUB1 in aneuploid HNSCC and lung cancer cell lines. Cancer Lett. 139:183-187.

23. Gemma, A., et al. 2000. Somatic mutation of the hBUB1 mitotic checkpoint gene in primary lung cancer. Genes Chromosomes Cancer. 29:213-218.

24. Haruki, N., et al. 2001. Molecular analysis of the mitotic checkpoint genes BUB1, BUBR1 and BUB3 in human lung cancers. Cancer Lett. 162:201-205.

25. Scolnick, D.M., and Halazonetis, T.D. 2000. Chfr defines a mitotic stress checkpoint that delays entry into metaphase. Nature. 406:430-435.

26. Bothos, J., Summers, M.K., Venere, M., Scolnick, D.M., and Halazonetis, T.D. 2003. The Chfr mitotic checkpoint protein functions with Ubc13-Mms2 to form Lys63-linked polyubiquitin chains. Oncogene. 22:7101-7107.

27. Chaturvedi, P., et al. 2002. Chfr regulates a mitotic stress pathway through its RING-finger domain with ubiquitin ligase activity. Cancer Res. 62:1797-1801.

28. Kang, D., Chen, J., Wong, J., and Fang, G. 2002. The checkpoint protein Chfr is a ligase that ubiquitinates Plk1 and inhibits Cdc2 at the G2 to M transition. J. Cell Biol. 156:249-259.

29. Yu, X., et al. 2005. Chfr is required for tumor suppression and Aurora A regulation. Nat. Genet. 37:401-406.

30. Privette, L.M., Weier, J.F., Nguyen, H.N., Yu, X., and Petty, E.M. 2008. Loss of CHFR in human mammary epithelial cells causes genomic instability by disrupting the mitotic spindle assembly checkpoint. Neoplasia. 10:643-652.

31. Privette, L.M., Gonzalez, M.E., Ding, L., Kleer, C.G., and Petty, E.M. 2007. Altered expression of the early mitotic checkpoint protein, CHFR, in breast cancers: implications for tumor suppression. Cancer Res. 67:6064-6074.

32. Michael, S., Trave, G., Ramu, C., Chica, C., and Gibson, T.J. 2008. Discovery of candidate KEN-box motifs using cell cycle keyword enrichment combined with native disorder prediction and motif conservation. Bioinformatics. 24:453-457.

33. Mizuno, K., et al. 2002. Aberrant hypermethylation of the CHFR prophase checkpoint gene in human lung cancers. Oncogene. 21:2328-2333.

34. Shibata, Y., et al. 2002. Chfr expression is downregulated by CPG island hypermethylation in esophageal cancer. Carcinogenesis. 23:1695-1699.

35. Satoh, A., et al. 2003. Epigenetic inactivation of CHFR and sensitivity to microtubule inhibitors in gastric cancer. Cancer Res. 63:8606-8613.

36. Corn, P.G., et al. 2003. Frequent hypermethylation of the 5' CpG island of the mitotic stress checkpoint gene $\mathrm{Chfr}$ in colorectal and non-small cell lung cancer. Carcinogenesis. 24:47-51.

37. Toyota, M., et al. 2003. Epigenetic inactivation of CHFR in human tumors. Proc. Natl. Acad. Sci.U. S. A. 100:7818-7823.

38. Mariatos, G., et al. 2003. Inactivating mutations targeting the chfr mitotic checkpoint gene in human lung cancer. Cancer Res. 63:7185-7189.

39. Baylin, S.B., and Herman, J.G. 2000. DNA hypermethylation in tumorigenesis: epigenetics joins genetics. Trends Genet. 16:168-174.

40. Koinuma, K., et al. 2006. Epigenetic silencing of AXIN2 in colorectal carcinoma with microsatellite instability. Oncogene. 25:139-146.

41. Bischoff, J.R., and Plowman, G.D. 1999. The Aurora/ Ipl1p kinase family: regulators of chromosome segregation and cytokinesis. Trends Cell Biol. 9:454-459.

42. Tanner, M.M., et al. 2000. Frequent amplification of chromosomal region $20 \mathrm{q} 12$-q13 in ovarian cancer. Clin. Cancer Res. 6:1833-1839.

43. Sen, S., Zhou, H., and White, R.A. 1997. A putative serine/threonine kinase encoding gene BTAK on chromosome $20 \mathrm{q} 13$ is amplified and overexpressed in human breast cancer cell lines. Oncogene. 14:2195-2200.

44. Zhou, H., et al. 1998. Tumour amplified kinase STK15/BTAK induces centrosome amplification, aneuploidy and transformation. Nat. Genet. 20:189-193.

45. Bischoff, J.R., et al. 1998. A homologue of Drosophila aurora kinase is oncogenic and amplified in 
human colorectal cancers. EMBO J. 17:3052-3065. 46. Meraldi, P., Honda, R., and Nigg, E.A. 2002. Aurora-A overexpression reveals tetraploidization as a major route to centrosome amplification in p53-/cells. EMBO J. 21:483-492.

47. Anand, S., Penrhyn-Lowe, S., and Venkitaraman, A.R. 2003. AURORA-A amplification overrides the mitotic spindle assembly checkpoint, inducing resistance to Taxol. Cancer Cell. 3:51-62.

48. Landen, C.N., Jr., et al. 2007. Overexpression of the centrosomal protein Aurora-A kinase is associated with poor prognosis in epithelial ovarian cancer patients. Clin. Cancer Res. 13 :4098-4104.

49. Li, D., et al. 2003. Overexpression of oncogenic STK15/BTAK/Aurora A kinase in human pancreatic cancer. Clin. Cancer Res. 9:991-997.

50. Tanaka, E., et al. 2005. The clinical significance of Aurora-A/STK15/BTAK expression in human esophageal squamous cell carcinoma. Clin. Cancer Res. 11:1827-1834.

51. Baker, S.M., et al. 1996. Involvement of mouse Mlh1 in DNA mismatch repair and meiotic crossing over. Nat. Genet. 13:336-342.

52. Edelmann, W., et al. 1996. Meiotic pachytene arrest in MLH1-deficient mice. Cell. 85:1125-1134.

53. Fukuda, T., Kondo, Y., and Nakagama, H. 2008. The anti-proliferative effects of the CHFR depend on the forkhead associated domain, but not E3 ligase activity mediated by ring finger domain. PLOS ONE. 3:e1776

54. Brandes, J.C., van Engeland, M., Wouters, K.A., Weijenberg, M.P., and Herman, J.G. 2005. CHFR promoter hypermethylation in colon cancer correlates with the microsatellite instability phenotype. Carcinogenesis. 26:1152-1156.

55. Nakagawa, H., et al. 2001. Age-related hypermethylation of the 5 ' region of MLH1 in normal colonic mucosa is associated with microsatelliteunstable colorectal cancer development. Cancer Res. 61:6991-6995

56. Morioka, Y., et al. 2006. Aberrant methylation of the CHFR gene is frequently detected in non-invasive colorectal cancer. Anticancer Res. 26:4267-4270.

57. Modrich, P. 1997. Strand-specific mismatch repair in mammalian cells. J. Biol. Chem. 272:24727-24730.

58. Duckett, D.R., et al. 1996. Human MutSalpha recognizes damaged DNA base pairs containing O6-methylguanine, O4-methylthymine, or the cisplatin-d(GpG) adduct. Proc. Natl. Acad. Sci. U. S. A. 93:6443-6447.

59. Gong, J.G., et al. 1999. The tyrosine kinase c-Abl regulates $\mathrm{p} 73$ in apoptotic response to cisplatininduced DNA damage. Nature. 399:806-809.

60. Hickman, M.J., and Samson, L.D. 1999. Role of DNA mismatch repair and p53 in signaling induc- tion of apoptosis by alkylating agents. Proc. Natl. Acad. Sci. U. S. A. 96:10764-10769.

61. Duckett, D.R., Bronstein, S.M., Taya, Y., and Modrich, P. 1999. hMutSalpha- and hMutLalphadependent phosphorylation of p53 in response to DNA methylator damage. Proc. Natl. Acad. Sci.U.S. A. 96:12384-12388.

62. Brown, K.D., et al. 2003. The mismatch repair system is required for S-phase checkpoint activation. Nat. Genet. 33:80-84.

63. Yoshioka, K., Yoshioka, Y., and Hsieh, P. 2006. ATR kinase activation mediated by MutSalpha and MutLalpha in response to cytotoxic O6-methylguanine adducts. Mol. Cell. 22:501-510.

64. Giannini, G. et al. 2002. Human MRE11 is inactivated in mismatch repair-deficient cancers. $E M B O$ Rep. 3:248-254.

65. Koh, K.H., et al. 2005. Impaired nonhomologous end-joining in mismatch repair-deficient colon carcinomas. Lab. Invest. 85:1130-1138.

66. Ham, M.F., et al. 2006. Impairment of doublestrand breaks repair and aberrant splicing of ATM and MRE11 in leukemia-lymphoma cell lines with microsatellite instability. Cancer Sci. 97:226-234.

67. Avdievich, E., et al. 2008. Distinct effects of the recurrent Mlh1G67R mutation on MMR functions, cancer, and meiosis. Proc. Natl. Acad. Sci. U. S. A. 105:4247-4252.

68. Jin, D.Y., Spencer, F., and Jeang, K.T. 1998. Human $\mathrm{T}$ cell leukemia virus type 1 oncoprotein Tax targets the human mitotic checkpoint protein MAD1. Cell. 93:81-91.

69. Zou, H., McGarry, T.J., Bernal, T., and Kirschner, M.W. 1999. Identification of a vertebrate sister-chromatid separation inhibitor involved in transformation and tumorigenesis. Science. 285:418-422.

70. Erson, A.E., and Petty, E.M. 2004. CHFR-associated early G2/M checkpoint defects in breast cancer cells. Mol. Carcinog. 39:26-33.

71. Summers, M.K., Bothos, J., and Halazonetis, T.D. 2005. The CHFR mitotic checkpoint protein delays cell cycle progression by excluding Cyclin B1 from the nucleus. Oncogene. 24:2589-2598.

72. Shtivelman, E. 2003. Promotion of mitosis by activated protein kinase B after DNA damage involves polo-like kinase 1 and checkpoint protein CHFR. Mol. Cancer Res. 1:959-969.

73. Oh, Y.M., et al. 2009. Chfr is linked to tumour metastasis through the downregulation of HDAC1. Nat. Cell Biol. 11:295-302.

74. Vogelstein, B., et al. 1989. Allelotype of colorectal carcinomas. Science. 244:207-211.

75. Thiagalingam, S., et al. 2001. Mechanisms underlying losses of heterozygosity in human colorectal cancers. Proc. Natl. Acad. Sci. U. S. A. 98:2698-2702.
76. Kern, S.E., et al. 1989. Clinical and pathological associations with allelic loss in colorectal carcinoma [corrected]. JAMA. 261:3099-3103.

77. Fang, Y., et al. 2004. ATR functions as a gene dosagedependent tumor suppressor on a mismatch repairdeficient background. EMBO J. 23:3164-3174.

78. Hurley, P.J., and Bunz, F. 2007. ATM and ATR: components of an integrated circuit. Cell Cycle. 6:414-417.

79. Ribic, C.M., et al. 2003. Tumor microsatellite-instability status as a predictor of benefit from fluorouracil-based adjuvant chemotherapy for colon cancer. N. Engl. J. Med. 349:247-257.

80. Jover, R., et al. 2006. Mismatch repair status in the prediction of benefit from adjuvant fluorouracil chemotherapy in colorectal cancer. Gut. 55:848-855.

81. de Vos tot Nederveen Cappel, W.H., et al. 2004. Survival after adjuvant 5-FU treatment for stage III colon cancer in hereditary nonpolyposis colorectal cancer. Int. J. Cancer. 109:468-471.

82. Carethers, J.M., et al. 2004. Use of 5-fluorouracil and survival in patients with microsatellite-unstable colorectal cancer. Gastroenterology. 126:394-401.

83. Cunningham, J.M. et al. 2001. The frequency of hereditary defective mismatch repair in a prospective series of unselected colorectal carcinomas. Am. J. Hum. Genet. 69:780-790.

84. Ballman, K.V., Grill, D.E., Oberg, A.L., and Therneau, T.M. 2004. Faster cyclic loess: normalizing RNA arrays via linear models. Bioinformatics. 20:2778-2786.

85. Chu, T.M., Weir, B., and Wolfinger, R. 2002. A systematic statistical linear modeling approach to oligonucleotide array experiments. Math. Biosci. 176:35-51.

86. Glebov, O.K., et al. 2003. Distinguishing right from left colon by the pattern of gene expression. Cancer Epidemiol. Biomarkers Prev. 12:755-762.

87. Birkenkamp-Demtroder, K., et al. 2005. Differential gene expression in colon cancer of the caecum versus the sigmoid and rectosigmoid. Gut. 54:374-384

88. Agrawal, D., et al. 2002. Osteopontin identified as lead marker of colon cancer progression, using pooled sample expression profiling. J. Natl. Cancer. Inst. 94:513-521.

89. Jassam, N., Bell, S.M., Speirs, V., and Quirke, P. 2005. Loss of expression of oestrogen receptor beta in colon cancer and its association with Dukes' staging. Oncol. Rep. 14:17-21.

90. Wu, L., Beito, T., and Chen, J. 2008. Generation and characterization of novel monoclonal antibodies against human aurora-A. Hybridoma (Larchmt.). 27:313-318.

91. Debes, J.D., et al. 2005. p300 regulates androgen receptor-independent expression of prostate-specific antigen in prostate cancer cells treated chronically with interleukin-6. Cancer Res. 65:5965-5973. 